complications between the 2 groups. The rate of margin positivity did not significantly differ between pure laparosocopy (15.8\%) and the robotic-assisted procedure $(19.5 \%)$. Our conclusion was that pure laparoscopic extra-peritoneal radical prostatectomy is equivalent to the robotic-assisted procedure in a centre experienced in laparoscopic techniques.

The current review is a welcome addition to the comparative literature regarding the status of minimally-invasive techniques against the well-established gold standard of open surgery. Tooher et al., in their comprehensive review of this topic, concluded that any conclusions that can be drawn from these comparisons are limited by the nature of the available data (4). Well performed, randomized, controlled trials are urgently required to provide stronger evidence when comparing these techniques. Sufficient followup and the use of internationally validated measures of functional outcomes are essential.

\section{REFERENCES}

1. Rozet F, Jaffe J, Braud G, Harmon J, Cathelineau X, Barret E, et al.: A direct comparison of robotic assisted versus pure laparoscopic radical prostatectomy: a single institution experience. J Urol. 2007; 178: 47882.

2. Menon M, Shrivastava A, Tewari A: Laparoscopic radical prostatectomy: conventional and robotic. Urology. 2005; 66 (5 Suppl): 101-4.

3. Joseph JV, Vicente I, Madeb R, Erturk E, Patel HR: Robot-assisted vs pure laparoscopic radical prostatectomy: are there any differences? BJU Int. 2005; 96: 39-42.

4. Tooher R, Swindle P, Woo H, Miller J, Maddern G: Laparoscopic radical prostatectomy for localized prostate cancer: a systematic review of comparative studies. J Urol. 2006; 175: 2011-7.

Dr. François Rozet \& Dr. Gordon P. Smith Department of Urology, Institut Montsouris Université Paris Descartes 42, Bd Jourdan, 75014 Paris, France E-mail:francois.rozet@imm.fr

\title{
Systemic Treatment for Invasive Bladder Cancer: Neoadjuvant Chemotherapy and Laparoscopic Radical Cystectomy
}

To the Editor,

The standard treatment for invasive transitional cell carcinoma (TCC) is radical cystectomy (RC) with lymphadenectomy; however, defining adequate therapy in every patient with invasive TCC remains difficult, because multiple biologic behavior patterns can be found in this disease (1).

Laparoscopy has come forward in oncologic urologic surgery to reproduce traditional operations in the endoscopic environment in order to minimize morbidity without compromising cancer outcomes. Laparoscopic radical cystectomy (LRC) was conceived as a procedure that could actually diminishes the associated morbidity of RC, while maintaining the oncological objectives.

Quality indicators in RC are well established nowadays: Mortality should not be higher than 2\% 
to $4 \%$. Positive surgical margin rates should be lower than $10 \%$ overall and $15 \%$ in pT3 or pT4 and the median number of pelvic nodes retrieved in the lymphadenectomy should be 10-14 (2). Simultaneously, orthotopic neobladder has become a surgical standard that improved the quality of life of these patients (3).

The surgical technique for radical cystectomy has specific technical objectives that should be met in every case (2):

1. Complete bladder cancer resection even in locally advanced tumors.

2. Minimal blood loss with early vascular control of superior and inferior vesical arteries.

3. Complete pelvic lymph node dissection.

4. Avoidance of tumor cell spillage.

Nowadays, the best outcomes in bladder cancer therapeutics are probably obtained when there is radical cystectomy in a systemic treatment setting. Neoadjuvant treatment has shown interesting advantages in patients with bladder cancer because it offers $5 \%$ of survival and $14 \%$ decreased risk of associated disease mortality (1). One might argue that two third of the patients would be treated without any response and survival advantage may be outweighed by potential treatment morbidity, with an important number of patients receiving chemotherapy to reach the $5 \%$ benefit, however, selection of the population incorporated in the protocols should address this issue.

Adequate surgical endoscopic skill developed in the last two decades and advances accomplished in the management of pulmonary, cardiovascular and hemodynamic effects of pneumoperitoneum allows offering laparoscopy as a safe alternative for these patients and recent data (4). Furthermore, as LRC has been reported with perioperative and functional outcomes comparable with open surgery and adequate mid-term cancer control (5), combining neoadjuvant therapy and LRC, would add the benefits of each one, and perhaps offer a more effective treatment for patients with invasive bladder cancer: The objective would be oncological efficacy with less morbidity. Clinical protocols addressing results of this mentioned way of treatment would be responsible for final answers in this matter and this constitutes our proposal for laparoscopy teams and medical oncologist, to unite for a common objective.
At the beginning of our experience with LRC the main consideration for surgery in bladder carcinoma was the precarious health of this patient's population. Things have not changed much; Haber and Gill (6) have reported important percentages of smokers (65\%), hypertension (59\%) and cardiac disease (17\%) in there series of long term follow-up for LRC. Today, we know that physiological changes incurred as a result of pneumoperitoneum have minimal adverse effects in the majority of patients undergoing laparoscopic surgery; therefore, in the setting of systemic treatment, LRC might represents the low morbidity surgical option for the patient who had neoadjuvant therapy. Minimizing operative trauma becomes even more important for these patients. To open the path, there is need for clinical protocols incorporating these therapeutical options in order to address initially the morbidity and mortality while keeping in mind the oncological safety.

\section{Take Home Message}

The combination of two effective treatments -medical and surgical- would probably offer a great advantage to patients with invasive bladder cancer. Laparoscopic cystectomy might represent a low morbidity surgical option to patients who have previously received chemotherapy for invasive bladder carcinoma.

\section{Acknowledgement}

The Institut Mutualiste Montsouris has started a protocol on neoadjuvant chemotherapy and laparoscopic cystectomy, funded in part by a Clinical Research Grant from Oficina de Investigacion, Confederacion Americana de Urologia, CAU.

\section{REFERENCES}

1. Herr HW, Dotan Z, Donat SM, Bajorin DF: Defining optimal therapy for muscle invasive bladder cancer. J Urol. 2007; 177: 437-43. 
2. Herr HW, Smith JA, Montie JE: Standardization of radical cystectomy: time to count and be counted. BJU Int. 2004; 94: 481-2.

3. Hautmann RE, Volkmer BG, Schumacher MC, Gschwend JE, Studer UE: Long-term results of standard procedures in urology: the ileal neobladder. World J Urol. 2006; 24: 305-14.

4. O’Malley C, Cunningham AJ: Physiologic changes during laparoscopy. Anesthesiol Clin North America. 2001; 19: 1-19.
5. Haber GP, Crouzet S, Gill IS: Laparoscopic and robotic assisted radical cystectomy for bladder cancer: a critical analysis. Eur Urol. 2008; 54: 54-64.

6. Haber GP, Gill IS: Laparoscopic radical cystectomy for cancer: oncological outcomes at up to 5 years. BJU Int. 2007; 100: 137-42.

\section{Dr. Eric Barret, Dr. Rafael Sanchez-Salas} \& Dr. Guy Vallancien

Department of Urology, Institut Montsouris

Université Paris Descartes 42, Bd Jourdan, 75014

Paris, France

E-mail: eric.barret@imm.fr 